\title{
Simplified supersymmetry with sneutrino LSP at 8 TeV LHC
}

\author{
Jun Guo, ${ }^{a}$ Zhaofeng Kang, ${ }^{b}$ Jinmian Li, ${ }^{a}$ Tianjun $\mathrm{Li}^{a}$ and Yandong Liu \\ ${ }^{a}$ State Key Laboratory of Theoretical Physics, Institute of Theoretical Physics, \\ Chinese Academy of Sciences, Beijing 100190, P.R. China \\ ${ }^{b}$ Center for High-Energy Physics, Peking University, \\ Beijing, 100871, P. R. China \\ ${ }^{c}$ School of Physical Electronics, University of Electronic Science and Technology of China, \\ Chengdu 610054, P.R. China \\ E-mail: hustgj@itp.ac.cn, zhaofengkang@gmail.com, jmli@itp.ac.cn, \\ tli@itp.ac.cn, ydliu@itp.ac.cn
}

ABSTRACT: The current searches of supersymmetry (SUSY) are based on the neutralino lightest sparticle (LSP). In this article we instead focus on SUSY with sneutrino LSP. It is well motivated in many contexts, especially in which sneutrino services as a dark matter candidate. We first develop a simplified model, which contains the stop, chargino/neutralino and sneutrino, to describe the LHC phenomenologies of a large class of models with sneutrino LSP. Then we investigate bounds on the model using the SUSY searches at the $8 \mathrm{TeV}$ LHC. Strong exclusion limits are derived, e.g., masses of stop and chargino can be excluded up to about $900 \mathrm{GeV}$ and $550 \mathrm{GeV}$, respectively. We also propose optimizations for some searches without turning to higher energy and luminosity.

Keywords: Supersymmetry Phenomenology, Monte Carlo Simulations

ARXIV EPRINT: 1312.2821 


\section{Contents}

1 Introduction and motivation $\quad 1$

2 Simplified model for SUSY with sneutrino LSP 2

2.1 Simplified model 2

2.2 Decay topologies in the simplified model 4

3 Constraining the simplified model at $8 \mathrm{TeV}$ LHC $\quad 6$

$\begin{array}{lll}3.1 & \text { Event generation and testing procedures } & 6\end{array}$

$\begin{array}{ll}3.2 & \text { Bounds on chargino/sneutrino }\end{array}$

$\begin{array}{lll}3.3 & \text { Bounds on stop/sneutrino } & 9\end{array}$

3.3.1 Bounds from top2: $\widetilde{t} \rightarrow t \widetilde{\nu} \nu \quad 9$

3.3.2 Bounds from top4: $\tilde{t} \rightarrow$ bl $\tilde{\nu} \quad 11$

3.3.3 Bounds from top3: $\tilde{t} \rightarrow b \chi \rightarrow b l \tilde{\nu} \quad 12$

$\begin{array}{lll}\text { 3.3.4 } & m_{T 2} \text { distinguishes the three-body from two-body decays } & 13\end{array}$

4 Conclusions and discussion $\quad 13$

$\begin{array}{ll}\text { A The stop and higgsino decays } & 15\end{array}$

$\begin{array}{ll}\text { B Simulation and Validation } & 16\end{array}$

\section{Introduction and motivation}

After the discovery of the standard model (SM)-like Higgs boson, supersymmetry (SUSY), which provides the most attractive solution to the hierarchy problem related to the Higgs field, becomes more theoretically attractive. Generically, the supersymmetic SMs (SSM) predict three kinds of new particles (i.e., the super-partner of the SM members), the colored particles like stop, the electroweak particles like Higgsinos and sleptons, and the missed particles, i.e., those are neutral and sufficiently long-lived at the LHC. It is expected that the lightest sparticle (LSP) should come from the third kind, since it then can be a weakly interacting massive particle (WIMP) dark mater (DM) candidate [1]. The early LHC searches for SUSY focused on the colored particles because they have large production rates at a hadronic collider, but now search for the electroweak sparticles is gaining increasing interests. In either case, the LSP shall play a key role because it is the terminal of other superparticles decay, thus providing missing energy to suppress the huge QCD backgrounds.

The nature of LSP is also pretty relevant to SUSY search because it can affect the sparticle decay chain thus the characteristic signature. But this aspect is not stressed much. The reason is due to the fact that in the benchmark model, the minimal SSM (MSSM), the lightest neutrino $\chi_{1}$ is the unique viable WIMP DM candidate [2-4]. However, beyond 
it the lightest sneutrino $\widetilde{\nu}_{1}$ can also service as a good candidate [5-15]. In the MSSM $\widetilde{\nu}_{1}$ is a fully electroweak complex scalar $\widetilde{\nu}_{L}$, and thus $Z$ boson mediates so large DM-nucleon recoil [16] that it has been excluded by the DM direct detection experiments like LUX [17] (given a correct DM relic density). In the low scale seesaw models, which are well motivated to explain neutrino masses, the situation changes. In those models, right handed neutrino (RHN)-like singlets usually are introduced and the corresponding sneutrino-like states may either properly mix with the $\widetilde{\nu}_{L}$ or couple significantly to visible fields, thus forming a viable WIMP DM. This paper is devoted to investigate SUSY search in the case of a sneutrino rather than neutralino LSP.

The leptonic nature of sneutrino LSP will bring a significant difference, since in general it leads to multi-leptons in the final states of sparticle decay. ${ }^{1}$ Compared to the neutralino LSP scenario, it is thus more likely to expose SUSY at LHC. In particular, if $\widetilde{\nu}_{1}$ is flavour biased, sparticle decay will yield different amount of lepton flavours. This may enhance the sparticle discovery potential by requiring a specific lepton flavour tagging. There are previous works taking advantage of the leptonic nature to explore relevant signatures. For example, based on the supersymmetric inverse seesaw [6, 9-15], ref. [21] studied the trilepton plus missing $E_{T}$ signature from $C_{1}^{ \pm} \chi_{2}$ production. Again in this model, ref. [12] studied the signature of same sign dilepton plus jets and missing energy from gluino- and squark- pair as well as the squark-gluino associated productions. Other relevant studies, some of which studied the previous signatures earlier, can be found in [22-32]. But these signatures are fairly model dependent thus not representative signatures of SUSY with a sneutrino LSP. Based on the simplified model for this SUSY scenario, we find that the opposite-sign dilepton plus missing energy with or without $b$-jets do capture its most common collider feature. And the current SUSY searches have already imposed stringent constraints on it.

The paper is organized as following. In section II, we will develop the simplified model to describe a large class of supersymmetric models with a sneutrino LSP. We also discuss the decay topologies in the model. In section III we make the collider setup and investigate the bounds on the model in light of the current LHC searches. Possible optimizations are also briefly discussed. Section IV is the discussion and conclusion. Some necessary and complementary details are given in the appendices.

\section{Simplified model for SUSY with sneutrino LSP}

In this section we will first develop the simplified model for SUSY with sneutrino LSP and introduce the conventions. Then, we analyze the basic collider features of the model, including decay lifetime and topologies of the sparticles within the model.

\subsection{Simplified model}

We are at the position to make a simplified model for SUSY with sneutrino LSP, $\widetilde{\nu}$. On top of $\widetilde{\nu}$, the model should contain three other superpartners, the stop $\widetilde{t}$, chargino $C^{ \pm}$and

\footnotetext{
${ }^{1}$ In the text we will show the decay of interest is prompt. The resulting signatures are different to the signatures of long-lived charged sparticle or displaced vertex, which usually appear in the case of quite weak couplings between the sneutrino LSP and sparticles in the visible sector [18-20].
} 
neutralino $\chi$. They represent the colored sparticles and electroweak sparticles, respectively. In particular, $C / \chi$ are always relevant when we are studying the colored sparticle (like stop) decaying into the sneutrino LSP. In addition, they have been extensively searched at the present LHC (based on the ordinary SUSY with neutralino LSP) and thus we can avail ourself of the public data to constrain them here. In a word, they are the proper minimal field content for the simplified model. The relevant interactions are casted in the following effective Lagrangian

$$
\begin{aligned}
-\mathcal{L}_{\mathrm{e} f f}= & m_{\widetilde{t}}^{2}|\widetilde{t}|^{2}+m_{\widetilde{\nu}}^{2}|\widetilde{\nu}|^{2}+m_{C} \bar{C} C+\frac{1}{2} m_{\chi} \chi \chi \\
& +\left[\bar{C}^{-}\left(g_{L}^{b} P_{L}+g_{R}^{b} P_{R}\right) b^{-} \widetilde{t}^{-}+\bar{C}^{-}\left(g_{L}^{e} P_{L}+g_{R}^{e} P_{R}\right) e^{-\widetilde{\nu}+\text { h.c. }]}\right. \\
& +\left[\bar{\chi}\left(g_{L}^{t} P_{L}+g_{R}^{t} P_{R}\right) t \widetilde{t}^{*}+\bar{\chi}\left(g_{L}^{\nu} P_{L}+g_{R}^{\nu} P_{R}\right) \nu \widetilde{\nu}+\text { h.c. }\right]
\end{aligned}
$$

with flavor index of leptons implied. Couplings involving the gauge bosons are not written out explicitly, and they are relevant only when we are discussing the chargino/neutralino productions. The sneutrino is assumed to be complex, but sneutrino being real scalar will not affect our discussions much.

The lagrangian of the simplified model is not simple. It contains quite a few free parameters. The effective coupling constants $g_{L / R}$ can be expressed in terms of the gauge/Yukawa coupling constants and the mixing angles in the stop and chargino sectors, etc. For instance, in the supersymmetric inverse seesaw models the sneutrino LSP DM may be a highly complex scalar, thus dominated by the singlets [14]. Then we can further simplify the model by working in the Higgsino-limit with $C^{-}=\left(\widetilde{H}_{d}^{-},\left(\widetilde{H}_{u}^{+}\right)^{\dagger}\right)^{T}$, where the couplings can be derived from the term in the superpotential, $y_{\nu} L H_{u} N$ :

$$
g_{L}^{e}=1, \quad g_{R}^{e}=0 ; \quad g_{L}^{\nu}=1, \quad g_{R}^{\nu}=0 .
$$

Similar chiral structure appears in the wino-limit. ${ }^{2}$ Note that in such limits the two degenerate neutralinos in eq. (2.1) pair up to form a Dirac fermion. In the collider search the concrete values of coupling constants are not important, except that branching ratios are of concern. Thus in various chiral limits we simply set the corresponding $g_{L / R}$ to be 1 or $0 .{ }^{3}$ Different chiral structures will lead to similar results, if we do not rely on the angular distributions of final states. We have more comments on this point in appendix B.

To end up this section, we would like to add some comments on the applicability of the simplified model in eq. (2.1). Firstly, in the non-thermal gravitino dark matter scenario, where gravitino gains correct relic density via the left-handed sneutrino NLSP later decay [37-39], sneutrino actually behaves as the LSP at colliders. Thus, such a SUSY scenario can be described by the simplified model. Next, in fact we do not need so large $g^{e, \nu}$ to make the sneutrino DM thermal. We only require it to ensure chargino dominantly

\footnotetext{
${ }^{2}$ This limit happens in the supersymmetric low-scale type-I seesaw model, in which $\widetilde{\nu}_{1}$ is a well mixture of $\widetilde{\nu}_{L}$ and $\widetilde{N}$, the right-handed sneutrino [5-8].

${ }^{3}$ This assumption is justified in the thermal $\widetilde{\nu}_{1}$ scenario, where it should couple to the visible sector with large couplings to annihilate away effectively. But if $\widetilde{N}$ has extra interactions $[11,24,33-36]$, it may break down.
} 

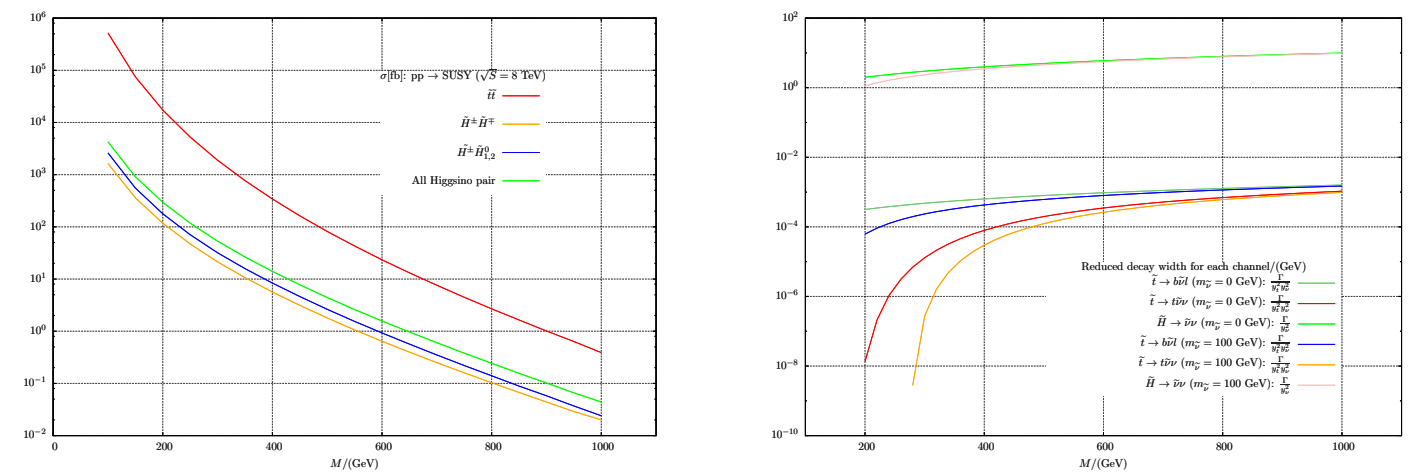

Figure 1. Left: production cross sections of stop pair, $\tilde{H}^{ \pm} \tilde{H}^{0}$ and $\tilde{H}^{ \pm} \tilde{H}^{\mp}$. Right: decays of stop/Higgsino. In top3 and top4, we set $m_{\chi}=1.1 m_{\tilde{t}}$ and $m_{C}=1.5 m_{\tilde{t}}$, respectively.

decays into $\widetilde{\nu}+e$ rather than into $\chi+W^{*}$. In this sense non-thermal sneutrino DM [40, 41] also may be described. Finally, $g^{t}$, etc., should be sufficiently large so that $\widetilde{t} / C / \chi$ decay promptly at the LHC.

To examine the second and third points aforementioned, we perform analytical calculations of the relevant decay rates and cast the complicated analytical expressions in appendix A. For illustration, we consider the right-handed stop/sneutrino and Higgsinolimit. First, to make chargino dominantly decays into sneutrino, one may need

$$
y_{\nu} \gtrsim \sqrt{\frac{32 \pi \Gamma_{C \rightarrow \chi W^{*}}}{\left(m_{\tilde{\chi}}^{2}-m_{\widetilde{\nu}}^{2}\right)^{2} / m_{\tilde{\chi}}^{3}}} .
$$

For example, for $m_{\widetilde{\nu}}=100 \mathrm{GeV}$ and $m_{\chi}=0.99 m_{C}$, we need $y_{\nu} \gtrsim 10^{-5}$; While as the degeneracy decrease slightly, says $m_{\chi}=0.95 m_{C}$, we need a much larger $y_{\nu} \gtrsim 10^{-3}$. In the thermal sneutrino DM scenario where $y_{\nu} \sim 1$ and therefore the non-gauge decay tends to be dominant, in particular for sizable $C-\chi$ degeneracy. Second, the traveling distances of stop, etc., are estimated as:

$$
c \tau= \begin{cases}\frac{1.975 \times 10^{-16}}{\Gamma_{R} y_{t}^{2} y_{\nu}^{2}}, & \tilde{t} \rightarrow t \nu \tilde{\nu}, b l \tilde{\nu}, \\ \frac{1.975 \times 10^{-16}}{\Gamma_{R} y_{\nu}^{2}}, & \tilde{H} \rightarrow \nu \tilde{\nu} .\end{cases}
$$

From figure 1 we see that as long as $y_{\nu} \gtrsim 10^{-5}\left(y_{\nu} \gtrsim 10^{-7}\right)$, stop (chargino) will three-body (two-body) decay promptly at the LHC.

\subsection{Decay topologies in the simplified model}

In the simplified model, the decay chains of $\widetilde{t}$, etc., terminate at the sneutrino LSP. Along these chains with chargino propagator, charged leptons are produced and potential to present characteristic signatures at the LHC. While decays with neutralino propagator bring no substantial difference to the conventional SUSY signature.

Before heading towards the main intent of this subsection, analyzing the decay topologies of $\widetilde{t}$ and $C, \chi$, we first show their production cross sections at the LHC. Stop, a colored 

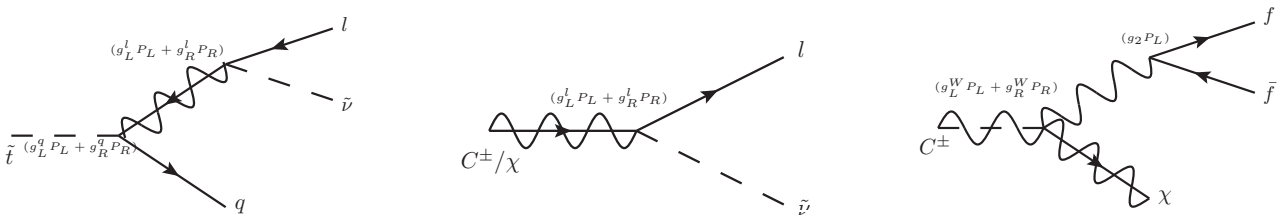

Figure 2. Decay Processes.

sparticle, has a large cross section of pair production. While productions of the electroweak sparticles $C / \chi$ depend on their ingredients. If $\chi$ is a $\mathrm{S} U(2)_{L}$ singlet, such as bino or singlino in the NMSSM, its direct production will be suppressed. However, when $C / \chi$ are in the Higgsino-limit or wino-limit, the $C$ and $\chi$ associated production will dominate over others. We show the numerical results, calculated by Prospino2 [42-44], in figure 1.

We now proceed to discuss the decay topologies. Evidently, the complete list is hard to be exhausted, because the decays heavily depend on the mass hierarchies. We thus only concentrate on the typical scenarios, which can be seen in the Feymann diagrams figure 2. If stop is so heavy, says owing to the relatively heavy SM-like Higgs boson, that it decouples from the simplified model, then we will have to probe SUSY in this scenario via the $C / \chi$ productions and decays (into sneutrino). Three decay topologies are generated

$$
\begin{aligned}
p p & \rightarrow C \bar{C} \rightarrow l^{ \pm} l^{\mp}+E_{T}^{\text {miss }} \\
& \rightarrow C^{ \pm} \chi \rightarrow l^{ \pm}+E_{T}^{\text {miss }} \\
& \rightarrow \chi \chi \rightarrow E_{T}^{\text {miss }}
\end{aligned}
$$

The present LHC searches are only sensitive to the first topology as will be shown later. If stop is relatively light, it will have a large production rate and give additional visible particles (such as the top or bottom quark) along the decay chains mediated by $C / \chi$. Basically, the stop decay topologies are classified into four categories:

Top1: neutralino is the next-to LSP (NLSP), with chargino decoupled. Moreover, stop is heavy enough so that $\chi$ is on-shell. Evidently, we can not distinguish this case to the ordinary case, i.e., the stop pair production with $\widetilde{t} \rightarrow t+E_{T}^{\text {miss }}$.

Top2: stop instead is the NLSP, and then $\chi$ is off-shell, i.e., the decay is three-body which means that the top quark of top2 is softened relative to that of top1. As a consequence, it is more difficult to hunt the stop than in the ordinary scenario. However, based on the different kinematic properties of final states between two body and three body decay as we will discuss in detail later, the scenarios can in principle be distinguished from the neutralino LSP case, if some signal events are observed at future collider.

Top3\&4: corresponding to top1\&2, these two are got by replacing $\chi$ with $C$. By virtue of the hard leptons in the final states, top $3 \& 4$ have better discovery prospects.

Based on these decay topologies, in the following section we will derive the current bounds on the simplified model by explaining the relevant SUSY searches at the $8 \mathrm{TeV}$ LHC. 


\begin{tabular}{|c|c|c|}
\hline Number & Reference & Sensitive Channel \\
1 & {$[49]$} & $\tilde{t} \rightarrow b l \tilde{\nu}$ \\
2 & {$[50]$} & $\tilde{t} \rightarrow b l \tilde{\nu}$ \\
3 & {$[51]$} & $\tilde{t} \rightarrow t \nu \tilde{\nu}$ \\
4 & {$[52]$} & $\tilde{t} \rightarrow t \nu \tilde{\nu}$ \\
5 & {$[53]$} & $\tilde{t} \rightarrow t \nu \tilde{\nu}, \tilde{t} \rightarrow b \tau \tilde{\nu}$ \\
6 & {$[54]$} & $C^{ \pm} C^{\mp} \rightarrow 2 \times l \tilde{\nu}$ \\
7 & {$[55]$} & $\tilde{t} \rightarrow b \tau \tilde{\nu}, C^{ \pm} C^{\mp} \rightarrow 2 \times \tau \tilde{\nu}$ \\
8 & {$[56]$} & $C^{ \pm} \chi \rightarrow \tau \nu 2 \times \tilde{\nu}$ \\
9 & {$[57]$} & $C^{ \pm} \chi \rightarrow l \nu 2 \times \tilde{\nu}$ \\
\hline
\end{tabular}

Table 1. The LHC searches used in this paper, where $l$ denotes either electron or muon. The number assigned for each search will be used in our plots to show the corresponding search which gives the strongest bound.

\section{Constraining the simplified model at $8 \mathrm{TeV}$ LHC}

In this section we derive the bounds on chargino/stop in terms of the decay topologies given previously. We also make comments on the possible optimizations of some searches for the corresponding signatures.

\subsection{Event generation and testing procedures}

In this paper events are generated by MadGraph5 [45], where Pythia6 [46] and PGS [47] have been packed to implement parton shower, hadronization and detector simulation. For detector simulation with PGS, we take the $b$-tagging efficiency $70 \%$, with $c$-mistag and lightjet mis-tag rates $20 \%$ and $10 \%$, respectively. Efficiency of tau-tagging is involved [48], and we simply take the "medium" jet discrimination quality for tau-tagging, whose efficiency is $40 \%$ (We neglect the mis-tag rate for non- $\tau$ jets, which is quite low, $\sim 1 \%$ ). For later convenience, we list the tau decay branching ratios:

$$
\begin{array}{ll}
\operatorname{Br}\left(\tau \rightarrow e \nu_{e} \nu_{\tau}\right)=17.9 \%, & \operatorname{Br}\left(\tau \rightarrow \mu \nu_{\mu} \nu_{\tau}\right)=17.4 \%, \\
\operatorname{Br}(\text { hadronic 1-prong decay })=49.5 \%, & \operatorname{Br}(\text { hadronic 3-prong decay })=15.2 \% .
\end{array}
$$

The current LHC searches that have sensitivity to the decay topologies in the simplified model are shown in table 1 . In the simulation we only consider one specific chirality structure, e.g., $\tilde{t}_{R} \rightarrow b_{L} \bar{e}_{L} \tilde{\nu}_{R}$ (All decays are prompt and have unit branching ratio). We have explicitly checked that changing the chirality can affect the distribution of events merely at the level of uncertainty. It thus allows us to use the same cuts used in the experiment analysis, to get the number of events in each signal region. In top2(4) we fix $m_{\chi}\left(m_{C}\right)=1.1(1.5) m_{\tilde{t}}$, and different ratios only change the kinematic distributions of final states slightly. More detail of the event simulation and check the validations of our implementing the experimental searches are discussed in appendix B.

We briefly describe how to derive the bounds (see also ref. [58]). In each signal region $S_{i}$ a variable $R_{v i s}^{i} \equiv \frac{N_{N P}^{i}}{N_{\text {limit }}^{i}}$ is introduced. $N_{\text {limit }}^{i}$ and $N_{N P}^{i}$ respectively are the experimental 
upper limit and new physics contributed number of events. Then new physics with $N_{N P}^{i}$ will be excluded, as long as there exists any $R_{v i s}^{i}>1$. The ATLAS collaborator explicitly gave $N_{\text {limit }}$, but CMS only presented the observed number of events and expected number of background events and the uncertainty in each signal region, so we have to derive the 95\% C.L. $N_{\text {limit }}$ from these data. This can be done via the standard Bayesian procedure:

$$
\frac{1}{\mathcal{N}} \int_{0}^{N_{\text {limit }}} \mathcal{L}\left(n_{\text {obs }} \mid N_{s}, N_{b}, \sigma_{b}\right) P\left(N_{s}\right) d N_{s}=0.95
$$

where $\mathcal{N}=\int_{0}^{\infty} \mathcal{L}\left(n_{\text {obs }} \mid N_{s}, N_{b}, \sigma_{b}\right) P\left(N_{s}\right) d N_{s}$, with a uniform prior probability $P\left(N_{s}\right)$, is a normalisation factor. Taking into account the uncertainties of background and signal, we should take the following likelihood:

$$
\mathcal{L}\left(n_{\text {obs }} \mid N_{s}, N_{b}, \sigma_{b}\right)=\frac{1}{\sqrt{2 \pi \sigma_{s}^{2}} \sqrt{2 \pi \sigma_{b}^{2}}} \int_{-5 \sigma_{s}}^{5 \sigma_{s}} d s \int_{-5 \sigma_{b}}^{5 \sigma_{b}} d b P\left(n_{o b s} ; \mu\right) e^{\frac{d b^{2}}{2 \sigma_{b}^{2}}} e^{\frac{d s^{2}}{2 \sigma_{s}^{2}}}
$$

Practically, the probability function $P\left(n_{o b s} ; \mu\right)$ takes Possion distribution $\mu^{n_{o b s}} e^{-\mu} / n_{o b s}$ ! for smaller $n_{o b s} \leqslant 100$ while Gaussian $e^{\left(n_{o b s}-\mu\right)^{2} / 2 \mu} / \sqrt{2 \pi \mu}$ for larger $n_{o b s}>100$. Here the expectation value $\mu=N_{s}+d s+N_{b}+d b$, and we assume the error $\sigma_{s}=0.01 n_{s}$. The derived $N_{\text {limit }}$ from eq. (3.3) will not change much as long as the signal uncertainty is within a few tens of percent, see appendix B.

\subsection{Bounds on chargino/sneutrino}

In this subsection we consider the bound on chargino/neutralino by decoupling stop. As have been discussed in section 2.1, we have three kinds of decay topologies, depending on the patterns of production. Among them, we do not discuss the one from $\chi \chi$ production, which gives missing energy only. It can be probed only if an initial visible particle like a hard jet is emitted, but the current mono-jet plus $E_{T}^{\text {miss }}$ search [59] is not able to impose a competitive bound, relative to the bounds from other decay topologies.

The only effective bound comes from $C^{ \pm} C^{\mp}$ pair production followed by $C^{ \pm} \rightarrow l \tilde{\nu}$. In what follows we will discuss two cases with $l=e / \mu$ and $\tau$, respectively.

- For the light lepton case, the decay topology gives rise to the signature of OppositeSign Dilepton (OSDL) plus large missing energy. The search for slepton pair production [54] can probe this signature. In this search, the leptonic $m_{T_{2}}$ variable [60-62]

$$
m_{T_{2}}=\min _{q_{T}}\left[\max \left(m_{T}\left(p_{T}^{l 1}, q_{T}\right), m_{T}\left(p_{T}^{l 2}, p_{T}^{\operatorname{miss}}-q_{T}\right)\right)\right]
$$

plays an important role to suppress the huge $t \bar{t}$ (and $W W$ as well) backgrounds, from which $m_{T_{2}}$ shows the sharp edge at $m_{W}$. While the edge of signal $m_{T_{2}} \simeq m_{C}$ is clearly larger than $m_{W}$, in particular for the heavier chargino. A large $E_{T}^{\text {miss }}$ may appear in the backgrounds, owing to the mis-measured momentum of jets or leptons. 

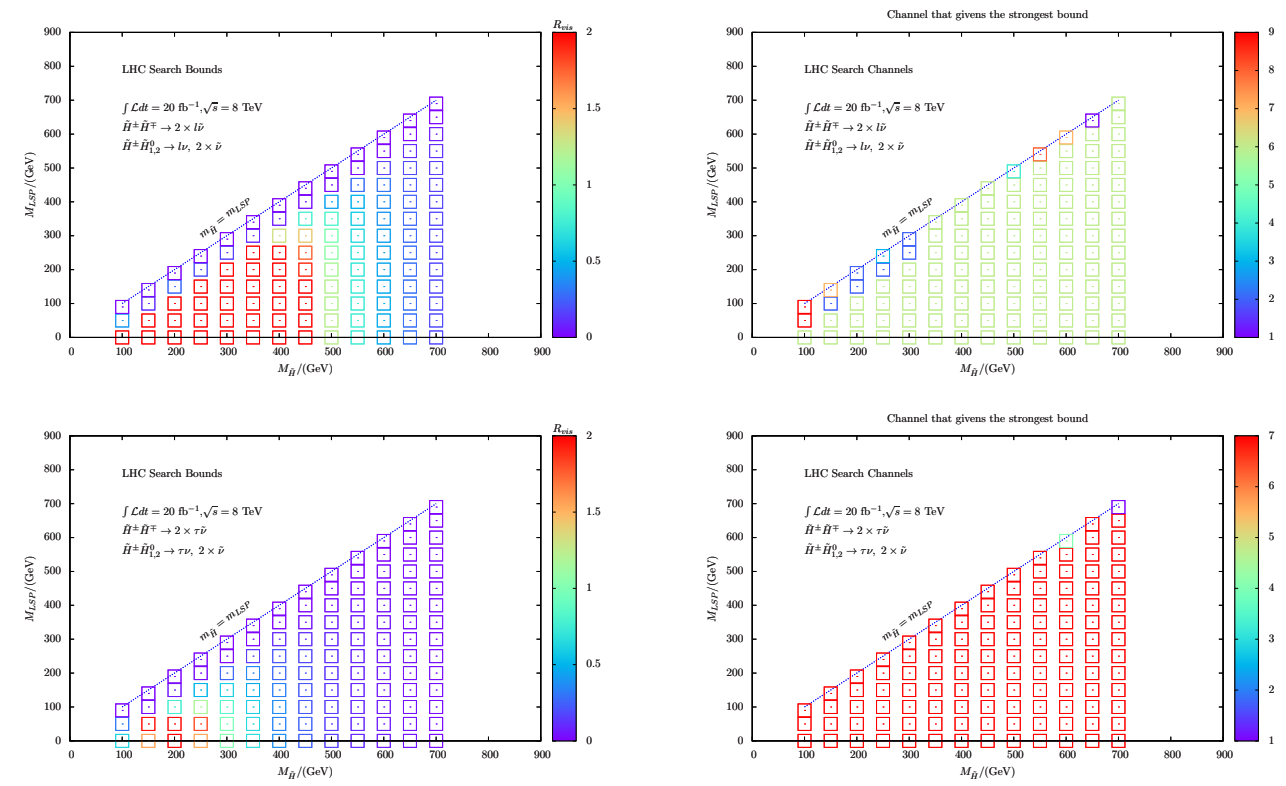

Figure 3. Bounds on charigno/neutrlaino in the Higgsino-limit (Both $\tilde{H}^{ \pm} \tilde{H}^{\mp}$ and $\tilde{H}^{ \pm} \tilde{H}_{1,2}^{0}$ productions are included). Top (bottom) left: the maximal $R_{v i s}$ (we have set any $R_{v i s}$ larger than 2 to be 2) among all search channels for final states with $\ell(\tau)$; top (bottom) right: the color coding of the channel which gives the most stringent bound, see table 1 . The same labels will be used throughout this paper.

In order to reduce that, the quality $E_{T}^{\text {miss,rel }}$ is introduced: ${ }^{4}$

$$
E_{T}^{\text {miss,rel }}=\left\{\begin{array}{ll}
E_{T}^{\text {miss }} & \text { if } \Delta \phi_{l, j} \geq \pi / 2 \\
E_{T}^{\text {miss }} \times \sin \Delta \phi_{l, j} & \text { if } \Delta \phi_{l, j}<\pi / 2
\end{array},\right.
$$

where $\Delta \phi_{l, j}$ is the azimuthal angle between the direction of $p_{T}^{\text {miss }}$ and its nearest lepton or jet. In addition, $Z$-veto is used to suppress the OSDL background from $Z$ decay.

The resulted constraints on charigno/sneutrino masses are similar to these on sleptons/neutralino masses in [54], but the exclusion limits is much higher by virtue of the larger cross section of chargino pair. The results are displayed in the upper panels of figure 3. From it one can see that mass of (the Higgsino-like) chargino smaller than $550 \mathrm{GeV}$ has been excluded, except that it degenerates with sneutrino, i.e., $m_{\tilde{H}}-m_{\tilde{\nu}} \lesssim 50 \mathrm{GeV}$.

- In the case of $\tau$ final state, e.g., the sneutrino LSP is dominated by the third generation of sneutrino, the constraint becomes comparatively weaker. Among three decay modes of the $\tau$-pair, the one with both hadronically decaying $\tau$ acquires the most stringent bound in terms of ref. [55]. It searches the signature of two tau-jets plus large $E_{T}^{\text {miss }}$, using similar ways to the above case to suppress backgrounds. For the

\footnotetext{
${ }^{4}$ Its variants are frequently used by many groups, in any context where $E_{T}^{\text {miss }}$ is a crucial kinematic cut. A good case in point is the search of $t \bar{t}$ plus $E_{T}^{\text {miss }}$ signature from stop pair production, as discussed later.
} 

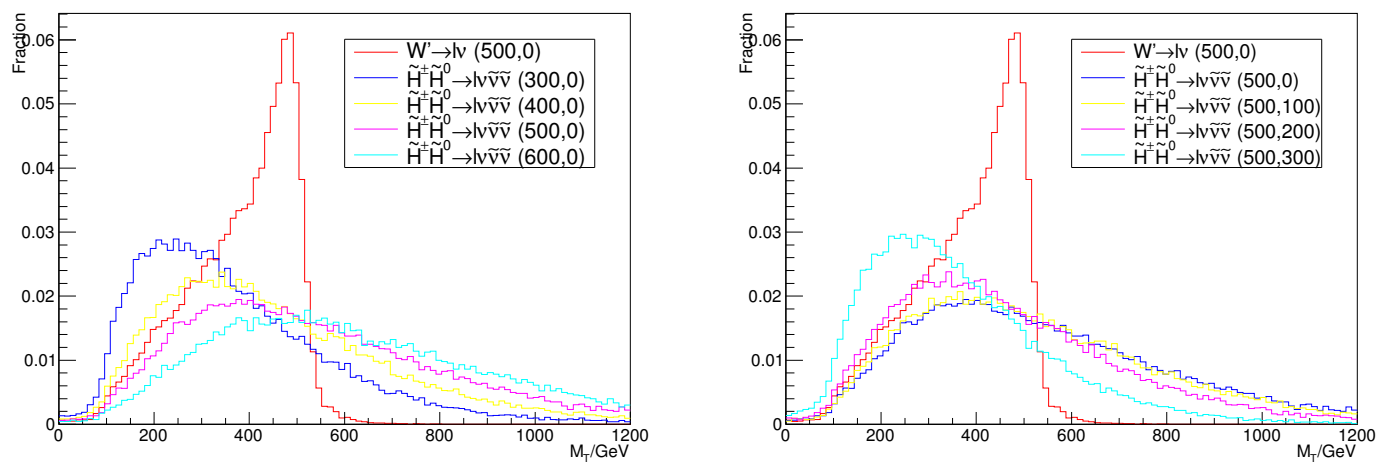

Figure 4. $M_{T}$ distribution for $W^{\prime}$ and Higgsino at detector level. The corresponding masses are given in the figure.

mode with both leptonically decaying $\tau$, the bound can be obtained as the $l=e / \mu$ case discussed before. We show the final bounds in the bottom panels of figure 3 . The exclusion is significantly weaker than the previous case, and $m_{C}$ can only be excluded up to $350 \mathrm{GeV}$ (for $m_{\tilde{\nu}} \lesssim 150 \mathrm{GeV}$ ). The reason, asides from the branching ratio suppression, is mainly blamed to the low $\tau$-tagging efficiency and the softer $m_{T 2}$.

To end up this section, we would like to make a comment on the sensitivity to the $C / \chi$ associated production. From figure 1 one can see that it has an even larger cross section than the chargino pair production, given $C / \chi$ in the Higgsino/wino-limit. Consider first the mono-lepton $(e / \mu)$ plus $E_{T}^{\text {miss }}$ signature, to which the only known relevant search by the CMS Collaboration [53] is not sensitive. The reason is simple: the CMS search heavily relies on the $M_{T}$ variable, which however has only been optimized for the heavy $W^{\prime}(\sim 1 \mathrm{TeV})$ search. Consequently, it fails in imposing bounds on the relatively light $C / \chi$, see figure 4. But $M_{T}$ is indeed a good variable to probe new physics, so hopefully we can improve the low $M_{T}$ region, by using additional cuts, to enhance sensitivity to this channel. The mono- $\tau$ channel, which gives rise to the mono-jet plus $E_{T}^{\text {miss }}$ signature, deserves special attention. Now, exclusion on new physics cross section from mono-jet search is merely about $100 \mathrm{fb}$ [59]. But jet flavor tagging may help much. Using the extrapolation from charm-tagging in the mono-jet search [59], the sensitivity is enhanced by about two magnitudes of order and reaches $\sigma>0.7 \mathrm{pb}$ (roughly corresponding to a bound $m_{C}>600 \mathrm{GeV}$ in the Higgsino limit).

\subsection{Bounds on stop/sneutrino}

Bounds on stop are complicated due to its rather rich decay topologies, top1-4. But top1 is identical to the ordinary SUSY case with stop pair production followed by $\widetilde{t} \rightarrow t \chi$, so in what follows we focus on top2-4.

\subsubsection{Bounds from top2: $\widetilde{t} \rightarrow t \widetilde{\nu} \nu$}

The final states of stop pair production in this channel contain a pair of top quarks plus missing energy, just the same as those of $\widetilde{t t^{*}} \rightarrow t \bar{t}+2 \chi$ in the conventional SUSY scenario. 

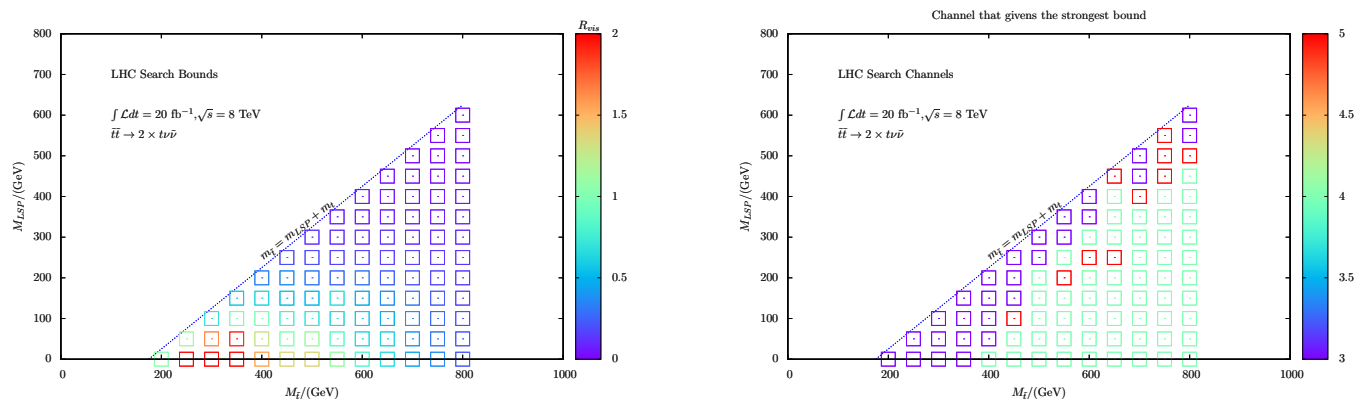

Figure 5. Bounds on stop/sneutrino masses from the $\tilde{t} \rightarrow t \nu \tilde{\nu}$ channel. Color scheme is the same as figure 3 .

As a matter of fact, the latter is expected to be the bulk signature in the supersymmetric models like MSSM and careful searches for it have been performed [51, 52]. Hence they can be used to well probe stop/sneutrino in our paper.

The most sensitive searching channel is different in different stop/sneutrino mass regions, depending on the efficiency of reconstructing the top quark. When their mass difference is relatively large $(\gtrsim 300 \mathrm{GeV})$, top quark from the heavy stop decay can be reconstructed rather effectively in a small cone and then search for the hadronic decaying stop pair, aided by a large $E_{T}^{\text {miss }}$, is able to give the strongest bound [52]. When the mass difference becomes smaller, top reconstruction becomes worse and hence the semileptonic stop pair, which gives one isolated hard lepton, will instead impose the strongest bound [51]. As the mass difference further decreases, namely it enters into the degenerate region, the signatures turn out to be almost indistinguishable from the $t \bar{t}$ background. Thus here we fail to make bounds on the stop/sneturino masses and should turn to other strategies [63-65]. But this topic is beyond the scope of this work.

The resulted bounds on stop and sneutrino masses are shown in figure 5 . It is seen that the exclusion limits have reached $m_{\tilde{t}} \lesssim 600 \mathrm{GeV}$ and $m_{\tilde{\nu}} \lesssim 100 \mathrm{GeV}$, but it is mildly weaker than the ordinary case $\tilde{t} \rightarrow t \chi$, which has reached the region $m_{\tilde{t}} \lesssim 700 \mathrm{GeV}$ and $m_{\tilde{\nu}} \lesssim 250 \mathrm{GeV}$. This is not surprising. In top2 stops are three-body decaying, which renders the kinematic distributions of the finals states more dispersed than these of the ordinary two-body decay. Especially, the missing energy is substantially softened. These features lead to the decreased sensitivity to the signature when we use data of searching stop pair with $\widetilde{t} \rightarrow t \chi$ to probe top2.

Actually, one can extend the current exclusion limit on the heavier stop ( $\gtrsim 600 \mathrm{GeV})$, by using HEPTopTagger [66] instead of the algorithm taken by experimentalists to tag the hadronic decaying tops.Even requiring the reconstructed top mass within a narrow window, $[150,200] \mathrm{GeV}$, HEPTopTagger can tag the top-jet with $p_{T}>200 \mathrm{GeV}$ efficiently $(\sim 37 \%)$. Thus it may help to improve our search for three-body decaying stop which produces relatively soft top. We have checked this for a mildly heavy stop with mass $600 \mathrm{GeV}$ (and $m_{\tilde{\nu}}=100 \mathrm{GeV}$ ), to find that the top-tagging efficiency still reaches $20.77 \%$ before the $p_{T}$ cut. 

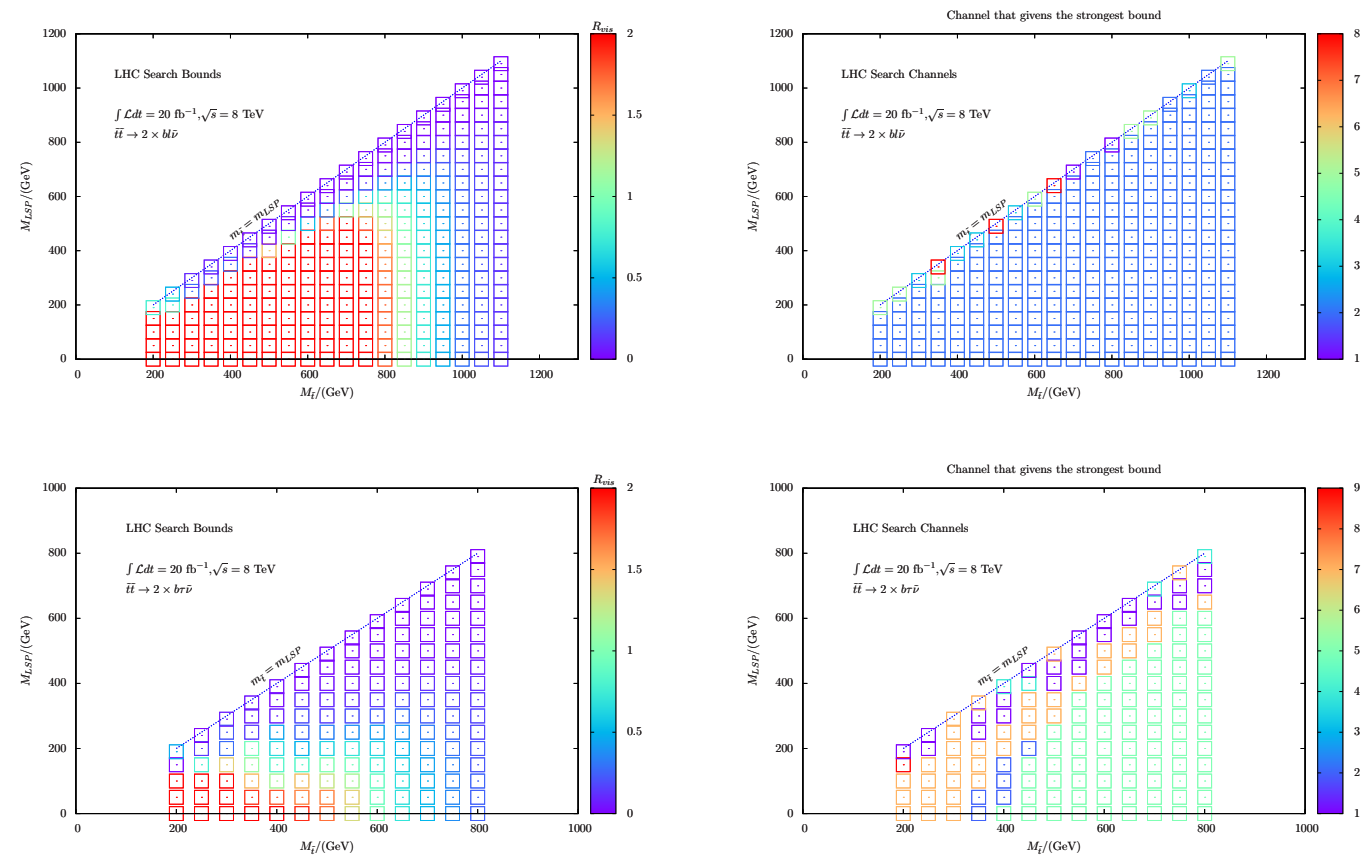

Figure 6. Bounds on stop/sneutrino masses. Top panels: from the $\tilde{t} \rightarrow b l \tilde{\nu}$ channel; bottom panels: from the $\tilde{t} \rightarrow b \tau \tilde{\nu}$ channel. Color scheme is the same as figure 3 .

\subsubsection{Bounds from top4: $\tilde{t} \rightarrow b l \tilde{\nu}$}

We postpone the more complicated case, top3, to the last subsubsection and here we focus on top4 first. As before, we respectively discuss two cases $l=e / \mu$ and $l=\tau$.

- For $l=e / \mu$, the final states contain two hard $b$-jets, two hard leptons with opposite sign (i.e., OSDL) and large missing energy. We can explore such a signature through the search for stop pair production followed by $\tilde{t} \rightarrow b C$ then $C \rightarrow W^{*} \chi$, which is another conventional bulk signature for stop [49]. This search is somewhat like the search of slepton pair production discussed previously, where the leptonic $m_{T 2}\left(\vec{p}_{T}^{11}, \vec{p}_{T}^{2}, p_{T}^{\text {miss }}\right)$ is an useful discriminator. And the presence of two hard $b$-jets further enhances the sensitivity to the signature. Note that the experimental cut on $m_{T 2}$ is relatively low, which benefits our case, where stop three-body decay leads to a softer $m_{T 2}$.

The bounds are displayed in the top panels of figure 6 . The bounds are fairly stringent, e.g., the stop mass have been excluded up to about $900 \mathrm{GeV}$, leaving only the degenerate region where the mass difference between $\tilde{t}$ and the LSP is smaller than $50 \mathrm{GeV}$. These exclusions are even more stringent than the exclusions for stop/neutralino in ref. [49], since there OSDL comes from the leptonic $W$ pair, suppressed by branching ratio. On top of that, here the leptons are directly produced from stop decay, so they can be quite hard.

- For $l=\tau$, searches depend on the decay of $\tau$. In the case of two hadronically decaying taus, the final states contain two tagged $b$ - and $\tau$-jets. Despite of no direct searches 
for such a signature, we can still probe them indirectly by means of the searches which tag two hadronically decaying taus or two $b$-jets. Similarly we can have sensitivity to the semi-leptonic decaying $\tau$ case. As for the two leptonic decaying taus case, the resulted signature actually is the same as the one discussed above. But here its cross section is suppressed by the squared $\tau$ leptonic branching ratio $\operatorname{Br}^{2}(\tau \rightarrow \ell \nu \nu) \simeq 0.1$.

The current bounds on this channel are shown in the bottom panels of figure 6 . The stop mass is excluded up to about $600 \mathrm{GeV}$ for $m_{\widetilde{\nu}}$ below $150 \mathrm{GeV}$. The bound on the heavier stop region is set by the CMS search [53] for signature of two $b$-jets, one lepton and $E_{T}^{\text {miss }}$. While at the lighter stop region with $m_{\tilde{t}}<350 \mathrm{GeV}$ and the degenerate region as well, the strongest bound comes from the search for final state with two tau-jets plus $E_{T}^{\text {miss }}[49]$ or two $b$-jets plus $E_{T}^{\text {miss }}$ [55]. And here the sneutrino mass has been excluded up to $200 \mathrm{GeV}$.

The bounds on this case will be further improved if tau is tagged out. Naively, the tau-richness in the final state is able to suppress the background by an order of magnitude, since tau in the background mainly comes from $W / Z$ boson decays, suppressed by the small branching ratios. Then, we may be able to push the bound on stop much above $600 \mathrm{GeV}$ in the semi-leptonic tau channel.

\subsubsection{Bounds from top3: $\tilde{t} \rightarrow b \chi \rightarrow b l \tilde{\nu}$}

Top3 and top4 share the same final states, but bounds on the former involve an extra parameter, mass of the chargino $m_{C}$. As a result, making a comprehensive bound becomes much more complicated, and we have to take several typical values of $m_{C}$ and then investigate the corresponding bounds on stop/sneutrino. Concretely, three typical cases are considered (we focus on $l=e / \mu$ case):

- The chargino mass is close to the sneutrino mass. In this case the lepton from chargino decay is too soft to be detected and then chargino behaves as a missing particle at the collider. The signature is two hard $b$-jets plus large $E_{T}^{\text {miss }}$, just the same as that of the ordinary channel $\tilde{t} \rightarrow b C^{ \pm}, C^{ \pm} \rightarrow W^{*} \chi$ with $m_{C} \simeq m_{\chi}$. From ref. [49] the stop mass has been excluded up to $600 \mathrm{GeV}$.

- Instead, the chargino mass is close to the stop mass, rendering the soft $b$-jets invisible. Evidently, this case is reduced to chargino pair production with a rescaled production cross section. Therefore, in the light of the bounds on charigno made in section. 3.2 and the production cross sections in figure 1, we estimate the bound on the stop mass, $\sim 900 \mathrm{GeV}$.

- Generically, the chargino mass is neither close to the sneutrino nor the stop mass. In this case the signature is identical to that of top4 and bounds again can be derived in the light of ref. [49]. We consider three representative examples for charigno mass: $m_{\tilde{\chi}^{ \pm}}=0.8 \times m_{\tilde{t}}, m_{\tilde{\chi}^{ \pm}}=0.5 \times m_{\tilde{t}}$ and $m_{\tilde{\chi}^{ \pm}}=0.2 \times m_{\tilde{t}}$. In each example, the strongest bound on stop mass is reached for a very light sneutrino. Concretely, it can be excluded up to about $850 \mathrm{GeV}, 850 \mathrm{GeV}$ and $600 \mathrm{GeV}$, respectively. The comparatively 

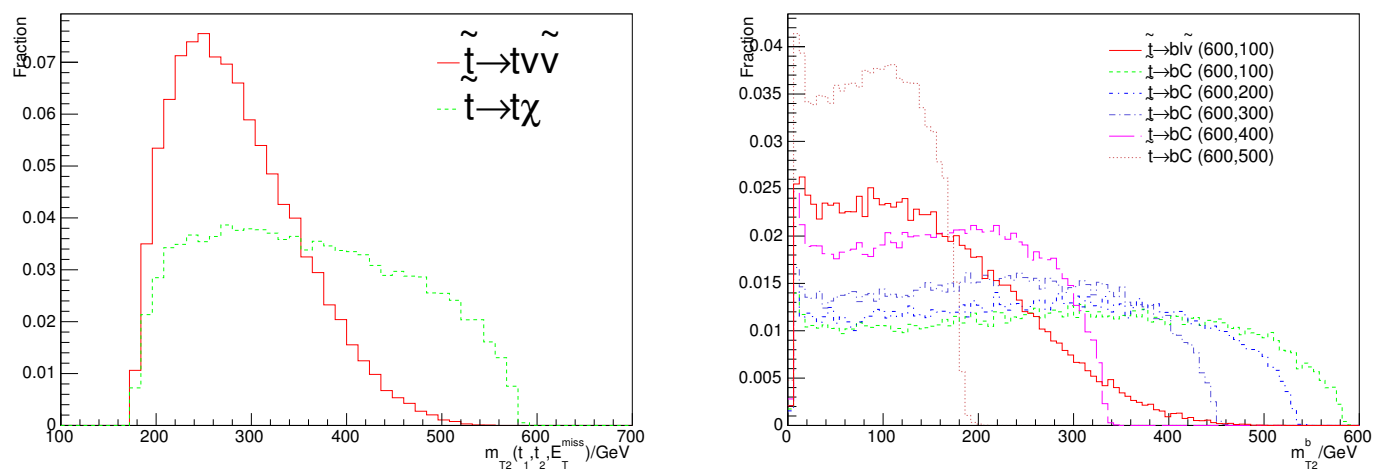

Figure 7. Comparisons of the $m_{T 2}$ distributions for two- and tree-body stop decays at parton level. Left: $m_{T 2}\left(t_{1}, t_{2}, p_{T}^{\text {miss }}\right)$ for decays mediated by neutralino. We take $m_{\tilde{t}}=600 \mathrm{GeV}$ and $m_{\mathrm{LSP}}=100 \mathrm{GeV}$. Right: $m_{T 2}^{b}$ for decays mediated by chargino. We take $m_{\tilde{t}}=600 \mathrm{GeV}$ and vary the charigno mass.

weaker bound on the third example is due to the smallness of $m_{T 2}\left(\vec{p}_{T}^{11}, \vec{p}_{T}^{22}, p_{T}^{\text {miss }}\right)$, which reflects the light chargino mass.

\subsection{4 $m_{T 2}$ distinguishes the three-body from two-body decays}

For a given process, signatures of three-body and two-body decays are the same with each other, e.g., stop pair production in top1 and top3, top2 and top4. Thus, (after the discovery of event excess) it is of interest to explore a kinematic variable to tell the difference. Specific to our article, $m_{T 2}$ is a good candidate.

First consider top1 (equivalent to $\widetilde{t} \rightarrow t \chi$ ) and top3. With the reconstructed top quarks, we can construct $m_{T 2}\left(t_{1}, t_{2}, p_{T}^{\text {miss }}\right)$. And we plot the distributions for top1 and top3 in the left panel of figure 7. Their shapes are clearly different: the curve for top1, namely for two-body decay, is much flatter and drops suddenly near $m_{\widetilde{t}}$; by contrast, the curve for top3, the three-body decay case, drops much earlier and faster. Alternatively, one can tell the difference by observing the property of curve at the tail. In two- and three-body decay they are convex and concave, respectively. Similar conclusion applies to top2 and top4, for which we use the modified $m_{T 2}$, i.e. $m_{T 2}^{b}[67]$ :

$$
m_{T 2}^{b}=\min \left\{\bigcup_{\vec{p}_{1}^{T}+\vec{p}_{2}^{T}=\vec{p}_{T}^{\text {miss }}+\vec{p}_{l 1}^{T}+\vec{p}_{l 2}^{t}} \max \left[M_{T}\left(\vec{p}_{b_{1}}, \vec{p}_{1}^{T}\right), M_{T}\left(\vec{p}_{b_{2}}, \vec{p}_{2}^{T}\right)\right]\right\} .
$$

where the leptons are also identified as missing energy and the bottom quarks are the only visible particles.

\section{Conclusions and discussion}

Due to a successful WIMP dark matter candidate, the lightest neutralino LSP is assumed to be the termination of sparticle cascade decays. It is the premise of most SUSY searches. But sneutrino LSP can also be a good thermal DM candidate in many supersymmetric 
SMs with low-scale seesaws. Thus it is well motivated to explore SUSY with sneutrino LSP. We construct a simplified model to describe their collider phenomenologies. Asides from the sneutrino, the model contains a stop, charigno and neutraino which can promptly decay into sneutrino at the LHC.

As a result of the leptonic nature of the sneutrino, leptons, associated with sneutrino, appear in the decay topologies of sparticles. This means that they may leave clear tracks at the collider. In fact, we find that the current SUSY searches at the LHC have already put strong bounds on them:

- For $C \rightarrow \ell \widetilde{\nu}$, the bound on charino/neutrino masses in the Higgsino-limit reaches $550 \mathrm{GeV}$, leaving the degenerate region with $m_{\tilde{H}}-m_{\widetilde{\nu}}<50 \mathrm{GeV}$ untouched. But for $C \rightarrow \tau \widetilde{\nu}$ the bound is substantially weakened, only excluding $m_{C} \lesssim 350 \mathrm{GeV}$ and $m_{\widetilde{\nu}} \lesssim 150 \mathrm{GeV}$. This is owing to both the inefficient $\tau$-tagging and the absence of specific searches.

- Mass of the NLSP stop has been excluded up to about $600 \mathrm{GeV}$ for $\widetilde{t} \rightarrow t \nu \widetilde{\nu}$ and up to about $900 \mathrm{GeV}$ for $\widetilde{t} \rightarrow b \ell \widetilde{\nu}$. In the former case, $m_{\widetilde{\nu}}$ is excluded up to about $100 \mathrm{GeV}$ while in the latter case only sneutrno in the degenerate region survives. For $\widetilde{t} \rightarrow b \tau \widetilde{\nu}$, compared to the $\tilde{t} \rightarrow b l \tilde{\nu}$ channel, the current searches have much weaker sensitivities not only for the heavier stop above $600 \mathrm{GeV}$ but also for the heavier sneutrino with mass above $200 \mathrm{GeV}$.

- If stop is not the NLSP, it will decay into neutralino or chargino first. The former case is just the same as the usual neutralino LSP scenario. The latter case can be divided into three categories, depending on the chargino mass. The stop mass have been excluded up to about $600 \mathrm{GeV} / 900 \mathrm{GeV}$ and $850 \mathrm{GeV}$ for chargino mass close to the sneutrino/stop mass and close to neither sneutrino nor stop mass.

We also briefly discuss possible optimizations for their searches without turning to the future LHC running.

What's more, the sneutrino LSP shows its features in many ways at collider which might enable us to distinguish it from neutralino LSP. Given the mass of mother particle, the first obvious result for three body decay with two invisible final states would be the softening of the visible particle which in turn will lead to softer missing energy. So, if we know the scale of a new particle production(e.g. from the initial state radiation [68]) and the scale of the particle decay, we might be able to tell which decay channel it is decaying through. Secondly, because the sneutrino LSP is carrying a lepton number, there will be more leptons produced in sneutrino LSP scenario than in neutralino LSP scenario. If those are charged leptons, then we may tell the natural of the LSP just according to the lepton number conservation. Thirdly, the width of three body decay depends on the mass of mediated particle. If the mediated particle is heavy enough, the produced new particle may have signature of displaced vertex. Further more, it may becomes long-lived particle, which is shown as charged track or R-hardron at collider. Finally, a more general method to distinguish those two scenarios is using the distribution of some featured kinematical variables. As we have briefly discussed in section 3.3.4, the distribution of $m_{T 2}$ variable 
can indeed distinguish $\tilde{t} \rightarrow t \nu \tilde{\nu}$ from $\tilde{t} \rightarrow t \chi$ and $\tilde{t} \rightarrow b l \tilde{\nu}$ from $\tilde{t} \rightarrow b C^{ \pm}$. We are hopping to find more effective discriminator, which we leave for the future study. In any case, the distinction is only meaningful after the discovery of a new signal.

\section{Acknowledgments}

This research was supported in part by the China Postdoctoral Science Foundation (No. 2012M521136) (ZK), and Natural Science Foundation of China under grant numbers 10821504, 11075194, 11135003, and 11275246 (TL).

\section{A The stop and higgsino decays}

In this appendix, we give the general expressions for the stop and chargino/neutralino decay widths. The Feymann diagrams are shown in figure 2. For the stop three-body decaying into sneutrino, we have

$$
\begin{aligned}
\Gamma= & \frac{1}{128 \pi} \frac{M_{\widetilde{t}}^{5}}{M_{\widetilde{\chi}^{4}}}\left(g_{L}^{q} g_{R}^{q}\left(g_{L}^{l}\right)^{2}+g_{L}^{q} g_{R}^{q}\left(g_{R}^{l}\right)^{2}\right) \widehat{f}_{3} \\
& +\frac{1}{256 \pi} \frac{M_{\widetilde{t}}^{5}}{M_{\widetilde{\chi}^{4}}}\left(\left(g_{L}^{q}\right)^{2}\left(g_{R}^{l}\right)^{2}+\left(g_{R}^{q}\right)^{2}\left(g_{L}^{l}\right)^{2}\right) \widehat{f}_{2}+\frac{1}{256 \pi} \frac{M_{\widetilde{t}}^{5}}{M_{\widetilde{\chi}^{4}}}\left(\left(g_{L}^{q}\right)^{2}\left(g_{L}^{l}\right)^{2}+\left(g_{R}^{q}\right)^{2}\left(g_{R}^{l}\right)^{2}\right) \widehat{f}_{1},
\end{aligned}
$$

where $\widehat{f}_{i}$ is the three-body final state phase space integral, given by

$$
\begin{aligned}
& \widehat{f}_{1}=\frac{m_{\tilde{\chi}}^{6}}{m_{\widetilde{t}}^{7}} \int_{m_{\widetilde{\nu}}^{2}}^{\left(m_{\tilde{t}}-m_{q}\right)^{2}} d P_{\tilde{\chi}}^{2} \frac{\sqrt{\left(\frac{m_{\tilde{t}}^{2}+P_{\tilde{\chi}}^{2}-m_{q}^{2}}{2 m_{\tilde{t}}}\right)^{2}-P_{\tilde{\chi}}^{2}}\left(P_{\tilde{\chi}}^{2}-m_{\widetilde{\nu}}^{2}\right)^{2}\left(m_{\tilde{t}}^{2}-m_{q}^{2}-P_{\widetilde{\chi}}^{2}\right)}{P_{\widetilde{\chi}}^{4}\left(P_{\tilde{\chi}}^{2}-m_{\tilde{\chi}}^{2}\right)^{2}}, \\
& \widehat{f_{2}}=\frac{m_{\tilde{\chi}}^{4}}{m_{\tilde{t}}^{5}} \int_{m_{\widetilde{\nu}}^{2}}^{\left(m_{\tilde{t}}-m_{q}\right)^{2}} d P_{\tilde{\chi}}^{2} \frac{\sqrt{\left(\frac{m_{\tilde{t}}^{2}+P_{\tilde{\chi}}^{2}-m_{q}^{2}}{2 m_{\tilde{t}}}\right)^{2}-P_{\tilde{\chi}}^{2}}\left(P_{\tilde{\chi}}^{2}-m_{\widetilde{\nu}}^{2}\right)^{2}\left(m_{\tilde{t}}^{2}-m_{q}^{2}-P_{\tilde{\chi}}^{2}\right)}{P_{\widetilde{\chi}}^{2}\left(P_{\tilde{\chi}}^{2}-m_{\tilde{\chi}}^{2}\right)^{2}}, \\
& \widehat{f_{3}}=\frac{m_{\tilde{\chi}}^{4}}{m_{\tilde{t}}^{5}} \int_{m_{\widetilde{\nu}}^{2}}^{\left(m_{\tilde{t}}-m_{q}\right)^{2}} d P_{\tilde{\chi}}^{2} \frac{m_{q} m_{\tilde{\chi}} \sqrt{\left(\frac{m_{\tilde{t}}^{2}+P_{\tilde{\chi}}^{2}-P_{\tilde{\chi}}^{2}}{2 m_{\tilde{t}}}\right)^{2}-P_{\tilde{\chi}}^{2}}\left(P_{\tilde{\chi}}^{2}-m_{\widetilde{\nu}}^{2}\right)^{2}}{P_{\widetilde{\chi}}^{2}\left(P_{\tilde{\chi}}^{2}-m_{\tilde{\chi}}^{2}\right)^{2}} .
\end{aligned}
$$

In eq. (A.1), one can easily track the origin of each term, back to the chiral structure of the vertices. We can express the effective coupling constants in terms of the fundamental coupling constants and mixing angles. For $\widetilde{t} \rightarrow t \nu \widetilde{\nu}$, we have [69]

$$
\begin{aligned}
g_{L}^{q} & =\frac{-2 \sqrt{2} i e}{3 c_{W}} N_{11} C_{\widetilde{t} R}+i y_{t} C_{\widetilde{t} L} N_{14}, \\
g_{R}^{q} & =\frac{i e}{\sqrt{2} s_{W} c_{w}} C_{\widetilde{t} L}\left(\frac{1}{3} N_{11} s_{W}+N_{12} c_{W}\right)+i y_{t} N_{14} C_{\widetilde{t} R}, \\
g_{L}^{l} & =\frac{i e}{\sqrt{2} s_{W} c_{w}} C_{\widetilde{\nu} L}\left(N_{11} s_{W}+N_{12} c_{W}\right)+i y_{\nu} N_{14} C_{\widetilde{\nu} R}, \quad g_{R}^{l}=i y_{\nu} N_{14} C_{\widetilde{\nu} L},
\end{aligned}
$$


where $C_{\widetilde{t} L}$ and $C_{\widetilde{t} R}$ respectively denote the left- and right-handed stop components, and $C_{\widetilde{\nu} L}$ and $C_{\widetilde{\nu} L}$ respectively denotes the left- and right-handed sneutrino components. Each of them has two parts, the bino/wino and Higgsino contributions. For $\widetilde{t} \rightarrow b l \widetilde{\nu}$,

$$
\begin{aligned}
g_{L}^{q} & =i y_{b} C_{\widetilde{t} L} U_{12}, & g_{R}^{q} & =\frac{i e}{s_{W}} C_{\widetilde{t} L} V_{11}+i y_{t} C_{\widetilde{t} R} V_{12}, \\
g_{L}^{l} & =\frac{i e}{s_{W}} C_{\widetilde{\nu} L} V_{11}+i y_{\nu} C_{\widetilde{\nu} L} V_{12}, & g_{R}^{l} & =i y_{l} C_{\widetilde{\nu} R} U_{12} .
\end{aligned}
$$

The two-body decay processes of chargino/neutralino are shown in the middle of figure 2. The decay width is calculated to be

$$
\Gamma=\frac{\left(g_{L}^{l}\right)^{2}+\left(g_{R}^{l}\right)^{2}}{32 \pi} \frac{\left(m_{C / \chi}^{2}-m_{\widetilde{\nu}}^{2}\right)^{2}}{m_{C / \chi}^{3}},
$$

where $g_{L}^{l}$ and $g_{R}^{l}$ come from the vertex $g_{L}^{l} P_{L}+g_{R}^{l} P_{R}$. For Higgsino (chargino) decay, $g_{L}^{l}$ and $g_{R}^{l}$ can be written as

$$
g_{L}^{l}=i y_{\nu} C_{\widetilde{\nu} R}\left(i y_{l} C_{\widetilde{\nu} L}\right), \quad g_{R}^{l}=i y_{\nu} C_{\widetilde{\nu} L}\left(i y_{\nu} C_{\widetilde{\nu} R}\right) .
$$

The three-body decay of chargino into neutralino and off-shell $W$ boson is shown in the right of figure 2 . The decay width is given by

$$
\Gamma \times \operatorname{Br}(e \nu)=\frac{1}{256 \pi^{3}} \frac{m_{C}^{5}}{m_{W}^{4}}\left(\left(g_{L}^{W}\right)^{2} g_{2}^{2}+\left(g_{R}^{W}\right)^{2} g_{2}^{2}\right) \widehat{f}_{1}+\frac{1}{128 \pi^{3}} \frac{m_{C}^{5}}{m_{W}^{4}}\left(g_{L}^{W} g_{R}^{W} g_{2}^{2}\right) \widehat{f}_{2},
$$

where the integral functions

$$
\begin{aligned}
& \widehat{f}_{1}=\frac{m_{W}^{4}}{m_{C}^{8}} \int_{0}^{\left(m_{C}-m_{\chi}\right)^{2}} d P_{W}^{2} \frac{\sqrt{k}}{\left(P_{W}^{2}-m_{W}^{2}\right)^{2}}\left(\frac{1}{2}\left(m_{C}^{2}-m_{\chi}^{2}\right)\left(m_{C}^{2}-m_{\chi}^{2}-P_{W}^{2}\right)-\frac{1}{6} k\right), \\
& \widehat{f}_{2}=\frac{m_{W}^{5}}{m_{C}^{7}} \int_{0}^{\left(m_{C}-m_{\chi}\right)^{2}} d P_{W}^{2} \frac{\sqrt{k}}{\left(P_{W}^{2}-m_{W}^{2}\right)^{2}} P_{W}^{2} .
\end{aligned}
$$

In the Higgsino-limit, $k$ and $g_{L}^{W}, g_{R}^{W}, g_{2}$ are explicitly given by

$$
\begin{aligned}
k & =m_{C}^{4}+m_{\chi}^{4}+P_{W}^{4}-2\left(m_{C}^{2} m_{\chi}^{2}+P_{W}^{2} m_{C}^{2}+P_{W}^{2} m_{\chi}^{2}\right), \\
g_{L}^{W} & =g_{R}^{W}=g_{2}=\frac{i e}{\sqrt{2} s_{W}} .
\end{aligned}
$$

\section{B Simulation and Validation}

In this appendix we give some details about the simulation for signatures used in the text. To ensure the validation of our simulation, we also presented the check for the usual SUSY searches in the relevant literatures.

Without loss (much) of generality, in the simulation only one specific chiral structure of the simplified model given in eq. (2.1) is considered. Concretely, we work in the Higgsino-limit and take $\tilde{t}_{L} \rightarrow t_{R} \tilde{H}_{u}^{0 *} \rightarrow t_{R}\left(\nu_{L} \tilde{\nu}_{R}\right)$ for top1/3, and $\tilde{t}_{R} \rightarrow b_{L} \tilde{H}_{u}^{+*} \rightarrow b_{L}\left(e_{L} \tilde{\nu}_{R}\right)$ 


\begin{tabular}{|c|c|c|c|c|}
\hline \multicolumn{5}{|c|}{ Low $\Delta \mathrm{M}$} \\
\hline Signal Region & $E_{T}^{\text {miss }}>100 \mathrm{GeV}$ & $E_{T}^{\text {miss }}>150 \mathrm{GeV}$ & $E_{T}^{\text {miss }}>200 \mathrm{GeV}$ & $E_{T}^{\text {miss }}>250 \mathrm{GeV}$ \\
\hline Background & $1662 \pm 203$ & $537 \pm 75$ & $180 \pm 28$ & $66 \pm 13$ \\
\hline Data & 1624 & 487 & 151 & 52 \\
\hline$N_{u p}$ & 473 & 189 & 59 & 29 \\
\hline \multicolumn{5}{|c|}{ High $\Delta \mathrm{M}$} \\
\hline Signal Region & $E_{T}^{\text {miss }}>100 \mathrm{GeV}$ & $E_{T}^{\text {miss }}>150 \mathrm{GeV}$ & $E_{T}^{\text {miss }}>200 \mathrm{GeV}$ & $E_{T}^{\text {miss }}>250 \mathrm{GeV}$ \\
\hline Background & $79 \pm 12$ & $38 \pm 7$ & $19 \pm 5$ & $9.9 \pm 2.7$ \\
\hline Data & 90 & 39 & 18 & 5 \\
\hline$N_{u p}$ & 46 & 22 & 15 & 7.1 \\
\hline
\end{tabular}

Table 2. CMS-PAS-SUS-13-011.
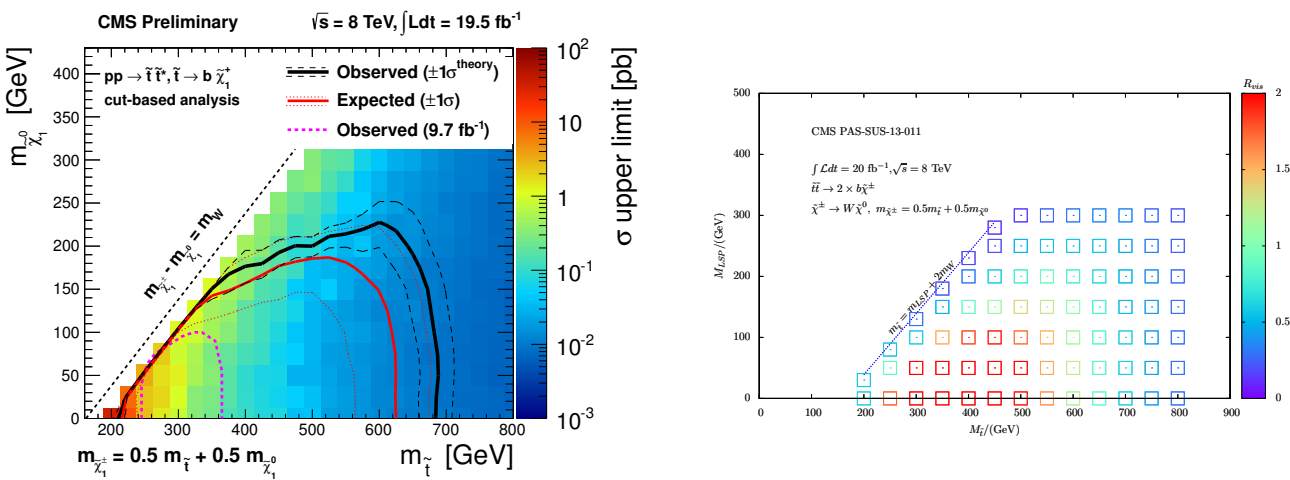

Figure 8. Left: experimental bounds on masses of stop and neutralino LSP given in [53]. Right: our check. Color scheme is the same as figure 3.

for top2/4. The UFO model files of the corresponding simplified model is generated by SARA4.0 [70]. Practically, we employ decays at the matrix element level so as to gain full helicity information in the final states, and find that decays in Pythia, which disregards helicity, leads to almost the same result. This justifies our simplifying treatment at the beginning.

Comments are in orders. Firstly, we do not discriminate electron and muon, because the difference between their detector efficiency is within the uncertainty in the simulation. Thus, as long as the flavour of the final state lepton is not concerned, which is the case in the current experimental analysis, our simulation is applicable to both flavour of the sneutrino LSP. Secondly, we emphasized that that the default PGS does not identify the sneutrino LSP as missing energy, and consequently it might be used to reconstruct jet. We have adapted the PGS for all flavors of sneutrinos being recognized as missing energy.

We now proceed to discuss the validation of our Monte Carlo simulation. We examined all the channels which give the strongest bounds in the text and found that the results are in accord with the LHC results, with the maximal deviation less than $50 \mathrm{GeV}$. For illustration, we show the check on ref. [53]. It searchs the final states with one isolated lepton, $b$-jets and missing energy. This search is sensitive to both $\tilde{t} \rightarrow t \nu \tilde{\nu}$ channel and $\tilde{t} \rightarrow b \tau \nu_{\tau}$ channel. In order to check both the Bayesian procedure used to estimate the upper limit and the cuts 
implemented, we generate the same process as that of ref. [53]. We scan the parameters $m_{\tilde{t}}$, $m_{\tilde{\chi}^{ \pm}}$and $m_{\mathrm{LSP}}$ with $m_{\tilde{\chi}_{1}^{ \pm}}=0.5 m_{\tilde{t}}+0.5 m_{\mathrm{LSP}}$ and then compare our bounds with theirs. The results are shown in figure 8 . In table 2, the upper limit of number of events for new physics contribution in each signal regions is given.

Open Access. This article is distributed under the terms of the Creative Commons Attribution License (CC-BY 4.0), which permits any use, distribution and reproduction in any medium, provided the original author(s) and source are credited.

\section{References}

[1] G. Bertone, D. Hooper and J. Silk, Particle dark matter: evidence, candidates and constraints, Phys. Rept. 405 (2005) 279 [hep-ph/0404175] [INSPIRE].

[2] H. Goldberg, Constraint on the photino mass from cosmology, Phys. Rev. Lett. 50 (1983) 1419 [Erratum ibid. 103 (2009) 099905] [INSPIRE].

[3] J.R. Ellis, J.S. Hagelin, D.V. Nanopoulos, K.A. Olive and M. Srednicki, Supersymmetric relics from the Big Bang, Nucl. Phys. B 238 (1984) 453 [inSPIRE].

[4] G. Jungman, M. Kamionkowski and K. Griest, Supersymmetric dark matter, Phys. Rept. 267 (1996) 195 [hep-ph/9506380] [INSPIRE].

[5] N. Arkani-Hamed, L.J. Hall, H. Murayama, D. Tucker-Smith and N. Weiner, Small neutrino masses from supersymmetry breaking, Phys. Rev. D 64 (2001) 115011 [hep-ph/0006312] [INSPIRE].

[6] C. Arina, F. Bazzocchi, N. Fornengo, J.C. Romao and J.W.F. Valle, Minimal supergravity sneutrino dark matter and inverse seesaw neutrino masses, Phys. Rev. Lett. 101 (2008) 161802 [arXiv:0806.3225] [INSPIRE].

[7] J. March-Russell, C. McCabe and M. McCullough, Neutrino-flavoured sneutrino dark matter, JHEP 03 (2010) 108 [arXiv:0911.4489] [INSPIRE].

[8] G. Bélanger, M. Kakizaki, E.K. Park, S. Kraml and A. Pukhov, Light mixed sneutrinos as thermal dark matter, JCAP 11 (2010) 017 [arXiv: 1008.0580] [INSPIRE].

[9] Z. Kang, J. Li, T. Li, T. Liu and J. Yang, Asymmetric sneutrino dark matter in the NMSSM with minimal inverse seesaw, arXiv:1102.5644 [INSPIRE].

[10] H. An, P.S.B. Dev, Y. Cai and R.N. Mohapatra, Sneutrino dark matter in gauged inverse seesaw models for neutrinos, Phys. Rev. Lett. 108 (2012) 081806 [arXiv:1110.1366] [INSPIRE].

[11] V. De Romeri and M. Hirsch, Sneutrino dark matter in low-scale seesaw scenarios, JHEP 12 (2012) 106 [arXiv:1209.3891] [INSPIRE].

[12] P.S. Bhupal Dev, S. Mondal, B. Mukhopadhyaya and S. Roy, Phenomenology of light sneutrino dark matter in cMSSM/mSUGRA with inverse seesaw, JHEP 09 (2012) 110 [arXiv: 1207.6542] [INSPIRE].

[13] K.-Y. Choi and O. Seto, Light Dirac right-handed sneutrino dark matter, Phys. Rev. D 88 (2013) 035005 [arXiv: 1305.4322] [INSPIRE]. 
[14] J. Guo, Z. Kang, T. Li and Y. Liu, Higgs boson mass and complex sneutrino dark matter in the supersymmetric inverse seesaw models, JHEP 02 (2014) 080 [arXiv:1311.3497] [INSPIRE].

[15] S. Banerjee, P.S.B. Dev, S. Mondal, B. Mukhopadhyaya and S. Roy, Invisible Higgs decay in a supersymmetric inverse seesaw model with light sneutrino dark matter, JHEP 10 (2013) 221 [arXiv: 1306.2143] [INSPIRE].

[16] T. Falk, K.A. Olive and M. Srednicki, Heavy sneutrinos as dark matter, Phys. Lett. B 339 (1994) 248 [hep-ph/9409270] [INSPIRE].

[17] LUX collaboration, D.S. Akerib et al., First results from the LUX dark matter experiment at the Sanford Underground Research Facility, Phys. Rev. Lett. 112 (2014) 091303 [arXiv: 1310.8214] [INSPIRE].

[18] B. Batell, S. Jung and C.E.M. Wagner, Very light charginos and Higgs decays, JHEP 12 (2013) 075 [arXiv: 1309.2297] [INSPIRE].

[19] D.G. Cerdeño, V. Martín-Lozano and O. Seto, Displaced vertices and long-lived charged particles in the NMSSM with right-handed sneutrinos, JHEP 05 (2014) 035 [arXiv: 1311.7260] [INSPIRE].

[20] C. Arina and M.E. Cabrera, Multi-lepton signatures at LHC from sneutrino dark matter, JHEP 04 (2014) 100 [arXiv: 1311.6549] [INSPIRE].

[21] S. Mondal, S. Biswas, P. Ghosh and S. Roy, Exploring novel correlations in trilepton channels at the LHC for the minimal supersymmetric inverse seesaw model, JHEP 05 (2012) 134 [arXiv: 1201.1556] [INSPIRE].

[22] Z. Thomas, D. Tucker-Smith and N. Weiner, Mixed sneutrinos, dark matter and the CERN LHC, Phys. Rev. D 77 (2008) 115015 [arXiv:0712.4146] [InSPIRE].

[23] A. Katz and B. Tweedie, Signals of a sneutrino (N)LSP at the LHC, Phys. Rev. D 81 (2010) 035012 [arXiv:0911.4132] [INSPIRE].

[24] H.-S. Lee and Y. Li, Identifying sneutrino dark matter: interplay between the LHC and direct search, Phys. Rev. D 84 (2011) 095003 [arXiv:1107. 0771] [INSPIRE].

[25] G. Bélanger, S. Kraml and A. Lessa, Light sneutrino dark matter at the LHC, JHEP 07 (2011) 083 [arXiv:1105.4878] [INSPIRE].

[26] A. de Gouvêa, S. Gopalakrishna and W. Porod, Stop decay into right-handed sneutrino LSP at hadron colliders, JHEP 11 (2006) 050 [hep-ph/0606296] [INSPIRE].

[27] C. Cheung, L.J. Hall and D. Pinner, Seesaw spectroscopy at colliders, arXiv:1103.3520 [INSPIRE].

[28] A. Das and N. Okada, Inverse seesaw neutrino signatures at the LHC and ILC, Phys. Rev. D 88 (2013) 113001 [arXiv: 1207.3734] [INSPIRE].

[29] M.A. Bernhardt, S.P. Das, H.K. Dreiner and S. Grab, Sneutrino as lightest supersymmetric particle in $B_{3}$ mSUGRA models and signals at the LHC, Phys. Rev.D 79 (2009) 035003 [arXiv:0810.3423] [INSPIRE].

[30] D. Choudhury, S.K. Gupta and B. Mukhopadhyaya, Right sneutrinos in a supergravity model and the signals of a stable stop at the Large Hadron Collider, Phys. Rev. D 78 (2008) 015023 [arXiv: 0804.3560] [INSPIRE]. 
[31] S.K. Gupta, B. Mukhopadhyaya and S.K. Rai, Right-chiral sneutrinos and long-lived staus: event characteristics at the large hadron collider, Phys. Rev. D 75 (2007) 075007 [hep-ph/0701063] [INSPIRE].

[32] A.T. Alan and S. Sultansoy, The 'Right' sneutrino as the LSP, J. Phys. G 30 (2004) 937 [hep-ph/0307143] [INSPIRE].

[33] D.G. Cerdeno and O. Seto, Right-handed sneutrino dark matter in the NMSSM, JCAP 08 (2009) 032 [arXiv: 0903.4677] [INSPIRE].

[34] P. Bandyopadhyay, E.J. Chun and J.-C. Park, Right-handed sneutrino dark matter in U(1)' seesaw models and its signatures at the LHC, JHEP 06 (2011) 129 [arXiv:1105.1652] [INSPIRE].

[35] M. Hirsch, W. Porod, L. Reichert and F. Staub, Phenomenology of the minimal supersymmetric $U(1)_{B-L} \times U(1)_{R}$ extension of the standard model, Phys. Rev. D 86 (2012) 093018 [arXiv: 1206.3516] [INSPIRE].

[36] F. Deppisch and A. Pilaftsis, Thermal right-handed sneutrino dark matter in the $F(D)$-term model of hybrid inflation, JHEP 10 (2008) 080 [arXiv:0808.0490] [INSPIRE].

[37] J.L. Feng, A. Rajaraman and F. Takayama, Superweakly interacting massive particles, Phys. Rev. Lett. 91 (2003) 011302 [hep-ph/0302215] [INSPIRE].

[38] J.L. Feng, S. Su and F. Takayama, Supergravity with a gravitino LSP, Phys. Rev. D 70 (2004) 075019 [hep-ph/0404231] [INSPIRE].

[39] Z. Kang and T. Li, Asymmetric origin for gravitino relic density in the hybrid gravity-gauge mediated supersymmetry breaking, JHEP 10 (2012) 150 [arXiv:1111.7313] [INSPIRE].

[40] T. Asaka, K. Ishiwata and T. Moroi, Right-handed sneutrino as cold dark matter, Phys. Rev. D 73 (2006) 051301 [hep-ph/0512118] [INSPIRE].

[41] S. Gopalakrishna, A. de Gouvêa and W. Porod, Right-handed sneutrinos as nonthermal dark matter, JCAP 05 (2006) 005 [hep-ph/0602027] [INSPIRE].

[42] W. Beenakker, R. Hopker and M. Spira, PROSPINO: a program for the production of supersymmetric particles in next-to-leading order QCD, hep-ph/9611232 [INSPIRE].

[43] W. Beenakker, M. Krämer, T. Plehn, M. Spira and P.M. Zerwas, Stop production at hadron colliders, Nucl. Phys. B 515 (1998) 3 [hep-ph/9710451] [INSPIRE].

[44] W. Beenakker et al., The Production of charginos/neutralinos and sleptons at hadron colliders, Phys. Rev. Lett. 83 (1999) 3780 [Erratum ibid. 100 (2008) 029901] [hep-ph/9906298] [INSPIRE].

[45] J. Alwall, M. Herquet, F. Maltoni, O. Mattelaer and T. Stelzer, MadGraph 5: going beyond, JHEP 06 (2011) 128 [arXiv:1106.0522] [INSPIRE].

[46] T. Sjöstrand, S. Mrenna and P.Z. Skands, PYTHIA 6.4 physics and manual, JHEP 05 (2006) 026 [hep-ph/0603175] [INSPIRE].

[47] J. Conway, http://www.physics.ucdavis.edu/ conway/research/software/pgs/pgs4-general.htm.

[48] ATLAS collaboration, Performance of the reconstruction and identification of hadronic $\tau$ decays with ATLAS, ATLAS-CONF-2011-152 (2011). 
[49] ATLAS collaboration, Search for direct third generation squark pair production in final states with missing transverse momentum and two b-jets in $\sqrt{s}=8 \mathrm{TeV}$ pp collisions with the ATLAS detector, ATLAS-CONF-2013-053 (2013).

[50] ATLAS collaboration, Search for direct top squark pair production in final states with two leptons in $\sqrt{s}=8 \mathrm{TeV}$ pp collisions using $20 \mathrm{fb}^{-1}$ of ATLAS data., ATLAS-CONF-2013-048 (2013).

[51] ATLAS collaboration, Search for direct top squark pair production in final states with one isolated lepton, jets and missing transverse momentum in $\sqrt{s}=8 \mathrm{TeV}$ pp collisions using $21 \mathrm{fb}^{-1}$ of ATLAS data, ATLAS-CONF-2013-037 (2013).

[52] ATLAS collaboration, Search for direct production of the top squark in the all-hadronic $t \bar{t}+$ etmiss final state in $21 \mathrm{fb}^{-1}$ of pp collisions at $\sqrt{s}=8 \mathrm{TeV}$ with the ATLAS detector, ATLAS-CONF-2013-024 (2013).

[53] CMS Collaboration, Search for top-squark pair production in the single lepton final state in pp collisions at $8 \mathrm{TeV}$, CMS-PAS-SUS-13-011 (2013).

[54] ATLAS collaboration, Search for direct-slepton and direct-chargino production in final states with two opposite-sign leptons, missing transverse momentum and no jets in 20/fb of pp collisions at $\sqrt{s}=8 \mathrm{TeV}$ with the ATLAS detector, ATLAS-CONF-2013-049 (2013).

[55] ATLAS collaboration, Search for electroweak production of supersymmetric particles in final states with at least two hadronically decaying taus and missing transverse momentum with the ATLAS detector in proton-proton collisions at $\sqrt{s}=8 \mathrm{TeV}$, ATLAS-CONF-2013-028 (2013).

[56] ATLAS collaboration, Search for supersymmetry in events with large missing transverse momentum, jets and at least one $\tau$ lepton in $21 \mathrm{fb}^{-1}$ of $\sqrt{\mathrm{s}}=8 \mathrm{TeV}$ proton-proton collision data with the ATLAS detector, ATLAS-CONF-2013-026 (2013).

[57] CMS Collaboration, Search for leptonic decays of $W^{\prime}$ bosons in pp collisions at $\sqrt{s}=8 \mathrm{TeV}$, CMS-PAS-EXO-12-060 (2012).

[58] T. Cheng, J. Li, T. Li and Q.-S. Yan, Natural NMSSM confronting with the LHC7-8, Phys. Rev. D 89 (2014) 015015 [arXiv: 1304.3182] [InSPIRE].

[59] ATLAS collaboration, Search for pair-produced top squarks decaying into a charm quark and the lightest neutralinos with $20.3 \mathrm{fb}^{-1}$ of pp collisions at $\sqrt{\mathrm{s}}=8 \mathrm{TeV}$ with the ATLAS detector at the LHC, ATLAS-CONF-2013-068 (2013).

[60] C.G. Lester and D.J. Summers, Measuring masses of semiinvisibly decaying particles pair produced at hadron colliders, Phys. Lett. B 463 (1999) 99 [hep-ph/9906349] [INSPIRE].

[61] A. Barr, C. Lester and P. Stephens, $m(T 2)$ : the truth behind the glamour, J. Phys. G 29 (2003) 2343 [hep-ph/0304226] [INSPIRE].

[62] H.-C. Cheng and Z. Han, Minimal kinematic constraints and m(T2), JHEP 12 (2008) 063 [arXiv:0810.5178] [INSPIRE].

[63] M.A. Ajaib, T. Li and Q. Shafi, Stop-neutralino coannihilation in the light of LHC, Phys. Rev. D 85 (2012) 055021 [arXiv:1111.4467] [INSPIRE].

[64] Z.-H. Yu, X.-J. Bi, Q.-S. Yan and P.-F. Yin, Detecting light stop pairs in coannihilation scenarios at the LHC, Phys. Rev. D 87 (2013) 055007 [arXiv:1211.2997] [INSPIRE].

[65] C. Han et al., Probing light Higgsinos in natural SUSY from monojet signals at the LHC, JHEP 02 (2014) 049 [arXiv: 1310.4274] [INSPIRE]. 
[66] T. Plehn, M. Spannowsky, M. Takeuchi and D. Zerwas, Stop reconstruction with tagged tops, JHEP 10 (2010) 078 [arXiv: 1006.2833] [INSPIRE].

[67] Y. Bai, H.-C. Cheng, J. Gallicchio and J. Gu, Stop the top background of the stop search, JHEP 07 (2012) 110 [arXiv: 1203.4813] [INSPIRE].

[68] J. Alwall, S. de Visscher and F. Maltoni, QCD radiation in the production of heavy colored particles at the LHC, JHEP 02 (2009) 017 [arXiv: 0810.5350] [INSPIRE].

[69] J. Rosiek, Complete set of Feynman rules for the MSSM: erratum, hep-ph/9511250 [INSPIRE].

[70] F. Staub, SARAH 4: a tool for (not only SUSY) model builders, Comput. Phys. Commun. 185 (2014) 1773 [arXiv:1309.7223] [INSPIRE]. 